
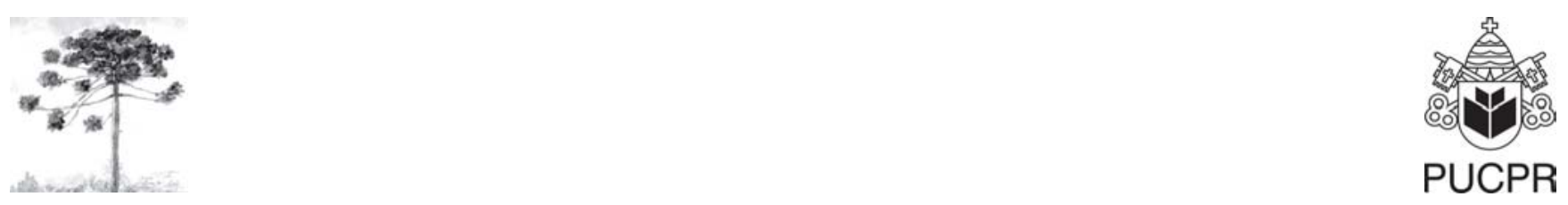

\title{
PREVALÊNCIA DE Dirofilaria immitis (LEIDY, 1856) EM CÃES DO CANIL MUNICIPAL DE GUARATUBA, PARANÁ, BRASIL
}

\author{
Prevalence of Dirofilaria immitis (Leidy, 1856) in dogs of Canil \\ Municipal Guaratuba, Parana, Brazil
}

\author{
Luiz Carlos Leite ${ }^{1}$, Mario Antonio Navarro-Silva ${ }^{2}$, Ennio Luz ${ }^{3}$, \\ Herminio de Paula Molinari ${ }^{4}$, Silvana Maris Círio ${ }^{5}$, Leide Parolim Marinoni ${ }^{6}$, \\ João Maria Ferraz Diniz ${ }^{7}$, Selene Círio Leite ${ }^{8}$, Diego Lunelli ${ }^{9}$, Willy Reiche Scalet ${ }^{10}$
}

\footnotetext{
${ }^{1}$ Professor Doutor. Curso de Medicina Veterinária - FURB-SC. Blumenau, SC - Brasil, e-mail: tudolica@pop.com.br

${ }^{2}$ Professor Doutor. Curso de Biologia. Depto. Entomologia - UFPR, Curitiba, PR - Brasil, e-mail: mnavarro@ufpr.br

${ }^{3}$ Professor Titular Livre Docente - Depto. Patologia Básica - UFPR, Curitiba, PR - Brasil, e-mail: sfzb@ufpr.br

${ }^{4}$ Professor Auxiliar - Curso de Medicina Veterinária - PUCPR, Curitiba, PR - Brasil, e-mail: hpm@onda.com.br

${ }^{5}$ Professora Doutora - Curso de Medicina Veterinária-CCAA - PUCPR, Curitiba, PR - Brasil, e-mail: silvana.cirio@pucpr.br

${ }^{6}$ Professora Livre Docente - Depto. Pediatria Hospital de Clínicas - UFPR, Curitiba, PR - Brasil, e-mail: clara.lara@ufpr.br

${ }^{7}$ Professor Adjunto Aposentado - Depto. Medicina Veterinária - UFPR, Curitiba, PR - Brasil, e-mail:1cmouraleite@pop.com.br

${ }^{8}$ Acadêmico - Curso de Medicina Veterinária - PUCPR, Curitiba, PR - Brasil, e-mail: seleneleite@gmail.com

${ }^{9}$ Acadêmico - Curso de Medicina Veterinária - PUCPR, Curitiba, PR - Brasil, e-mail: dilunelli@hotmail.com

${ }^{10}$ Acadêmico - Curso de Medicina Veterinária - UNIGUAÇUPR, União da Vitória, PR - Brasil, e-mail: willyscalet@yahoo.com.br
}

\section{Resumo}

A presente investigação permitiu determinar a prevalência de Dirofilaria immitis em cães alojados no Canil da Prefeitura Municipal de Guaratuba, Paraná, por meio de três diferentes técnicas hematológicas: a) Exame direto do sangue a fresco, b) Método de KNOT'T (1939) modificado, c) Canine Heartworm Antigen Test ${ }^{\mathbb{B}}$ (Snap Test). Foram avaliados 111 machos e 102 fêmeas, apreendidos e recolhidos pelo Serviço de Vigilância Municipal de Guaratuba entre maio de 2001 e dezembro de 2002. As amostras de sangue revelaram dois cães positivos para $D$. immitis. O primeiro reagiu de forma positiva aos três testes laboratoriais utilizados, enquanto o outro cão acusou positividade apenas no Snap Test, caracterizando dirofilariose oculta. A prevalência foi de $0,94 \%$ de cães infectados por Dirofilaria immitis, de um total de 213 animais avaliados.

Palavras-chave: Zoonoses; Dirofilaria immitis; Filariose; Cães. 


\begin{abstract}
The present investigation has an objective to determinate the prevalence of Dirofilaria immitis in dogs housed at the City of Guaratuba Municipal Kennel - Paraná, under three diferents haematologic technics: a) direct exam of fresh blood b) Method of KNOTT (1939) modified, c ) Canine Heartworm Antigen Test ${ }^{\circledR}$ (Snap Test). Was avaliated 111 males and 102 females, apprehended and withdrawn by Guaratuba Municipal Vigilance Service, between May 2001 and December 2002. The blood samples revelead two positive dogs for $\boldsymbol{D}$. immitis. One of them reacted by positive form at the three laboratory tests used, while the other dog accured positivity only in Snap Test characterizing hidden dirofilariasis. The prevalence was 0,94\% of dogs infected by D. immitis, than a total of 213 evaluated animals.
\end{abstract}

Keywords: Zoonosis; Dirofilaria immitis; Filariasis; Dogs.

\section{INTRODUÇÃO}

Dirofilaria immitis (Leidy, 1856) é um filarídeo parasita do ventrículo e átrio direitos do coração e artéria pulmonar de carnívoros domésticos, silvestres e de alguns animais domésticos como os cavalos. Conforme Ludlam et al. (1), o ciclo de vida inclui a passagem obrigatória das microfilárias de D. immitis pelos hospedeiros intermediários, os mosquitos culicídeos suscetíveis. A dirofilariose foi reportada no Brasil em diferentes graus de incidência por Kasai et al. (2); Labarthe et al. (3, 4), Ferreira et al. (5), Fernandes et al. (6), Suassuana et al. (7) e Reifur et al. (8). A forma cardiopulmonar é citada por Larsson et al. (9) e Theis (10) como a mais observada. Entretanto, o animal pode igualmente apresentar a forma subclínica da doença. Segundo Browne et al. (11) e Echeto et al. (12), o cão enquadra-se como hospedeiro significativo e estudos desenvolvidos por Atkins et al. (13) consideram o gato como hospedeiro atípico de Dirofilaria immitis. Robinson et al. (14) reportaram a importância da dirofilariose como zoonose ainda não estudada de forma satisfatória.

Takeuchi et al. (15) observaram que em seres humanos os parasitas raramente atingem a fase adulta. Shah (16), Flieder e Moran (17), Cavallazzi et al. (18), Oshiro et al. (19), Asimacopoulos et al. (20), Vital et al. (21) registraram o encontro de formas imaturas de $D$. immitis no interior de nódulos inseridos nos pulmões de pessoas infectadas, sendo estas estruturas freqüentemente confundidas com neoplasia por alguns estudiosos. No Brasil, é conhecido um único caso de dirofilariose cardíaca humana, atribuído à Dirofilaria magalhaesi Blanchard, 1896, registrada por De Magalhães (22) no ventrículo esquerdo do coração de uma criança.

Dada a importância do parasita e as implicações provocadas por dirofilariose em animais e seres humanos, o objetivo do presente trabalho foi direcionado na determinação da prevalência de Dirofilaria immitis em cães alojados no Canil Municipal de Guaratuba - PR, por meio de diferentes exames hematológicos.

\section{MATERIAIS E MÉTODOS}

\section{Área do estudo}

O trabalho foi desenvolvido no município de Guaratuba - PR, localizado em área litorânea do estado do Paraná, a 119 km de Curitiba, em uma planície peninsular arenosa, com uma das frentes situada na baía de Guaratuba a Noroeste, e outra frente ao Oceano Atlântico, a Sudeste. Possui $22 \mathrm{~km}$ de praias, altitude de 15 $\mathrm{m}$, latitude $-25^{\circ} 53^{\prime}$ 60' S e longitude - 48 34' 0" W. Sua área é de $1.328,480 \mathrm{~km}^{2}$ e a população total de 33.058 habitantes, sendo 27.257 indivíduos componentes da população urbana residente, conforme censo do IBGE (23). O município é dotado de estrutura de saneamento básico composta de rede de esgoto, abastecimento com água tratada e coleta de lixo atendendo respectivamente 5.425, 18.379 e 6724 domicílios permanentes e particulares. Dotado de extensão continental costeira e estuarina que abriga uma 
variedade de ambientes como a baía de Guaratuba, Serra do Mar, ilhas e manguezais (Figura 1). Em relação à fauna desta região da Mata Atlântica, de acordo com dados do Ipardes (24), é considerável o número de espécies de animais silvestres, relacionados como possíveis hospedeiros de Dirofilaria immitis, a exemplo do mão-pelada, gato-do-mato, furão, cachorro-do-mato, gato-mourisco e jaguatirica.

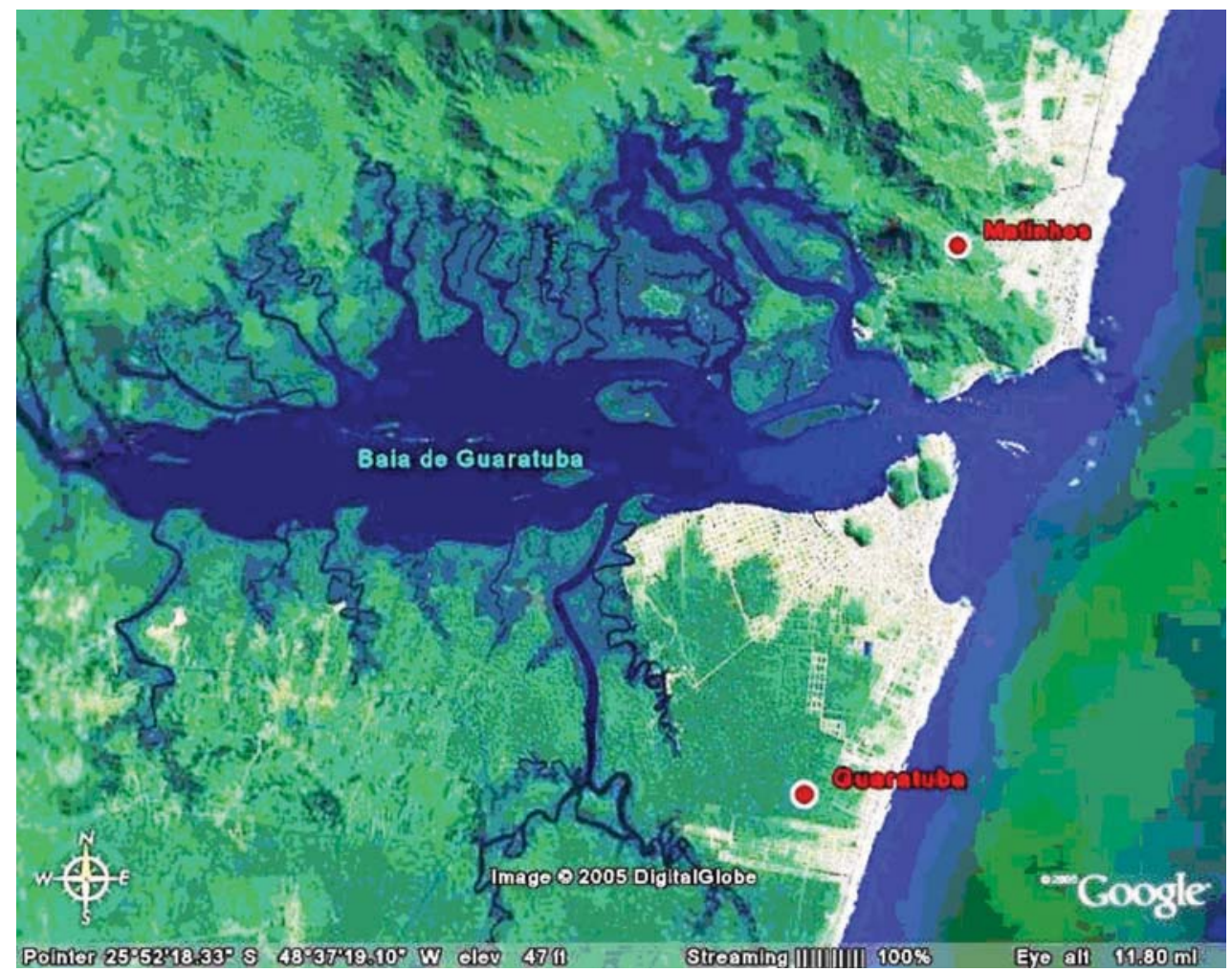

FIGURA 1 - Imagem de satélite (Google 2005) onde estão assinalados a baia de Guaratuba e os municípios de Guaratuba e Matinhos

\section{Metodologia}

O Canil Municipal situado em Guaratuba - PR foi previamente selecionado para as coletas e análise de sangue de cães errantes capturados, visando identificar a presença de microfilárias de Dirofilaria immitis.

Cães apreendidos pelo serviço de captura realizado pela Prefeitura de Guaratuba - PR, no período de maio de 2001 a dezembro de 2002, foram avaliados no presente trabalho. Não foram levados em consideração a raça, o sexo, a idade, o porte ou pelagem dos animais. Os animais foram submetidos individualmente a exames clínicos de rotina, por meio de inspeção direta da pelagem e da pele, estado nutricional, coloração de mucosas, cavidades naturais e da dentição, esta com a finalidade de determinação da idade aproximada, conforme Grandjean (25). De cada animal posicionado em estação ou decúbito lateral, contido por mordaça descartável, foi coletado $5 \mathrm{ml}$ de sangue em seringa descartável acoplada à agulha 25" X 8", por meio de punção direta na veia jugular, após prévia assepsia no local com álcool iodado, sendo as respectivas amostras identificadas individualmente, acondicionadas em frascos contendo anticoagulante EDTA $(0,03 \mathrm{ml}$ de Sal Sódico do Ácido Etilenodiaminotetracético), mantidas em refrigeração em caixa de isopor com gelo, enviadas ao Laboratório de Parasitologia Veterinária da PUCPR, armazenadas em refrigerador com temperatura de 2 a $4^{\circ} \mathrm{C}$ e examinadas em um prazo máximo de 24 horas após a coleta. Em seguida, foram realizados os seguintes exames de sangue nos cães para determinação da prevalência de Dirofilaria immitis: a) Exame direto do sangue fresco, b) Método de Knott (1939) modificado por Newton \& Wright (26), c) Snap Canine Heartworm Antigen Test Kit (IDEXX Laboratories). Mesmo aqueles cães desverminados anteriormente foram 
avaliados, uma vez que poderiam ser portadores de anticorpos circulantes contra Dirofilaria immitis. Cães com menos de seis meses de idade foram descartados da avaliação. Cada amostra de sangue foi submetida simultaneamente aos três métodos laboratoriais, como forma de aumentar a confiabilidade dos resultados.

\section{RESULTADOS}

Avaliou-se um total de 213 cães apreendidos pelo serviço de captura realizado

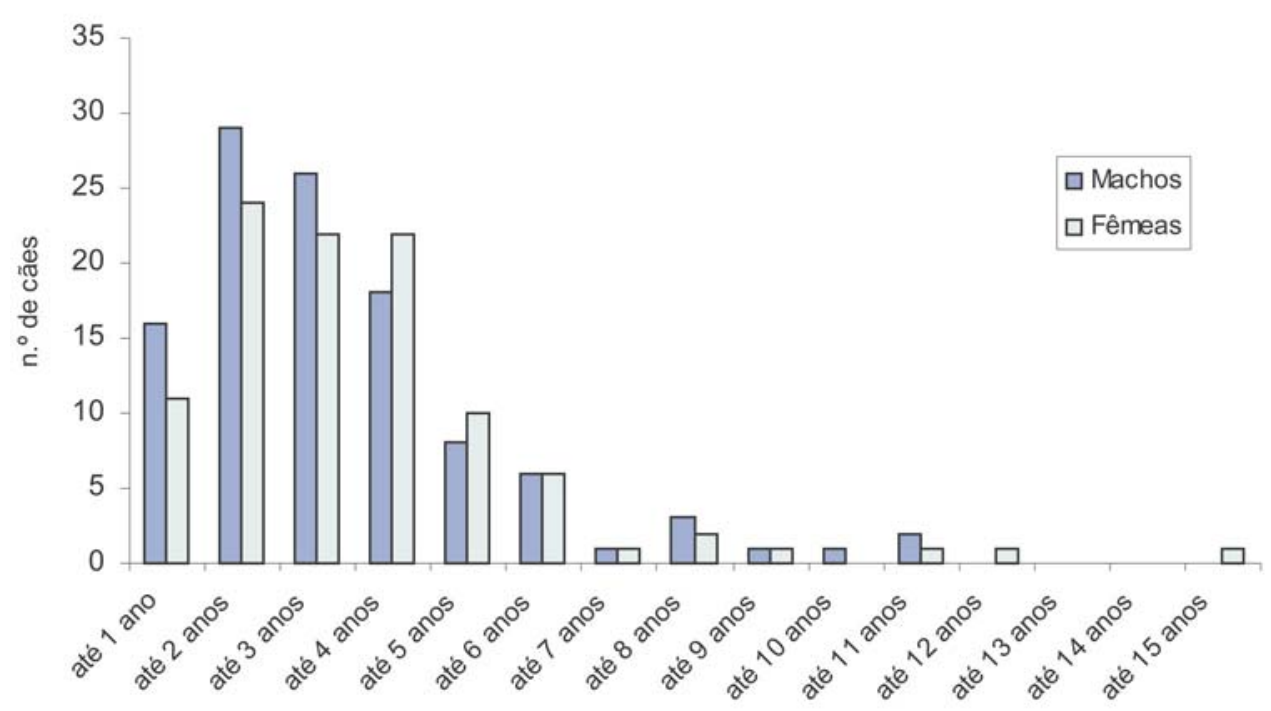

FIGURA 2 - Idade dos cães machos e fêmeas avaliados na pesquisa de microfilárias de Dirofilaria immitis, abrigados no Canil Municipal de Guaratuba, PR, no período entre maio de 2001 e dezembro de 2002

A maioria dos animais era de porte pequeno e médio, totalizando 90,6\% (Figura 3).

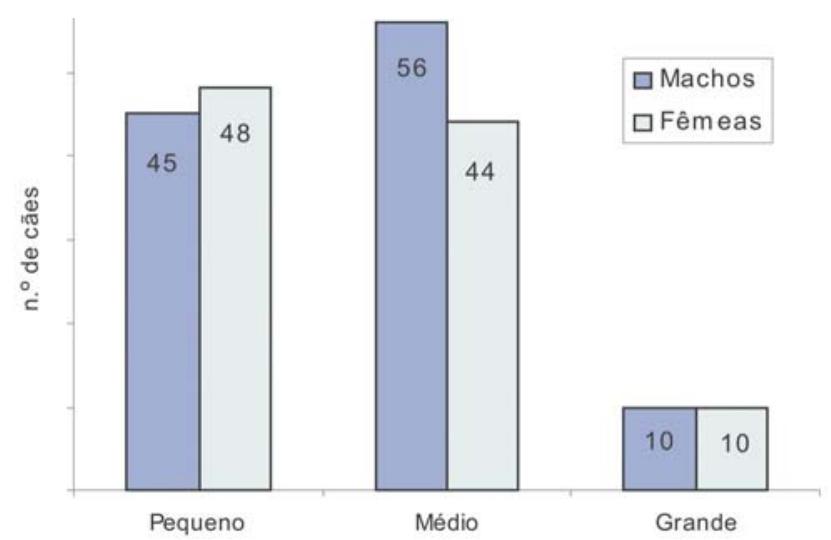

FIGURA 3 - Porte dos cães machos e fêmeas avaliados no

\footnotetext{
Canil Municipalde Guaratuba,PR, noperíodo
entre maio de 2001 e dezembro de 2002

Canil Municipalde Guaratuba, PR, noperíodo
entre maio de 2001 e dezembro de 2002
}

pela Prefeitura de Guaratuba - PR, no período de maio de 2001 a dezembro de 2002. Desse total, $111(52,1 \%)$ animais eram do sexo masculino e $102(47,9 \%)$ do sexo feminino. Os animais sem raça definida constituíram-se em maioria, compreendendo $92,9 \%$, sendo $7,1 \%$ divididos entre animais mestiços e com raça. A pelagem apresentou diversos padrões de coloração, sendo mais comuns bege, preto, tricolor e bicolor, somando $77,5 \%$.

As idades variaram entre um e quinze anos, prevalecendo os animais de um a quatro anos, 78,8\% do grupo avaliado (Figura 2).

Estud. Biol. 2007 jan/mar;29(66):73-79 
Prevalência de Dirofilaria immitis (Leidy, 1856) em cães do canil municipal de Guaratuba, Paraná, Brasil

TABELA 1 - Características fenotípicas e idade dos cães com testes positivos para Dirofilaria immitis detectados no Canil Municipal de Guaratuba (PR)

\begin{tabular}{llllllllll}
\hline Cães & Idade & Sexo & Porte & Raça & Pelagem & Data Coleta & V.D. & KNOTT & ELISA \\
\hline 01 & 06 Anos & M & Pequeno & S.R.D. & Dourada & $20 / 04 / 2002$ & + & + & + \\
02 & 04 Anos & M & Pequeno & S.R.D. & Preta & $20 / 07 / 2002$ & - & - & + \\
& & & & & & & & & \\
TOTAL & & & & & & & 01 & 01 & 02 \\
\hline
\end{tabular}

S.R.D. - Sem Raça Definida

M - Macho

V.D. - Teste de Visualização Direta (Gota Espessa)

KNOTT - Teste de Knott modificado

ELISA - Teste Imunoenzimático

\section{DISCUSSÃO E CONCLUSÕES}

A investigação da prevalência de Dirofilaria immitis detectou reduzido número de cães positivos. Em relação ao sexo dos animais estudados, um número maior de cães foi observado. A preferência por machos normalmente é relacionada à inexistência dos períodos de estro e gestação presentes nas fêmeas. Houve predomínio de espécimes sem raça definida, o que levou a uma variabilidade extrema nos itens como pelagem, coloração e porte. Normalmente os animais sem raça definida são acolhidos pela população de baixa renda devido à sua rusticidade, maior resistência em relação a doenças infecciosas e parasitárias, menor exigência de espaço para abrigo e menor exigência alimentar. Foram observados animais com idades superiores a dez anos, o que é muito significativo em relação à vida média de um cão. Os resultados percentuais obtidos na presente investigação $(0,94 \%)$ são menores que os registrados em 14 cães (5,4\%) para Dirofilaria immitis pelos testes de ELISA realizados no litoral do estado do Paraná (8), em Niterói e Rio de Janeiro, onde foram detectados $83(13,9 \%)$ cães microfilarêmicos e $44(7,9 \%)$ com dirofilariose oculta em amostras de sangue por meio de Teste de ELISA e de Knott modificado (3, 4), por Serrão et al. (27) em Itacoatiara (RJ) que analisaram 123 amostras também pelo teste de Knott modificado (22), encontrando microfilárias de Dirofilaria immitis em 39 (31,7\%) amostras. Índices superiores também foram reportados por Garcez et al. (28) em Salvaterra, Ilha de Marajó (PA), sendo constatada a positividade ao teste de ELISA em 48 amostras $(53,5 \%)$, de 90 avaliadas.
A densidade da população canina nas áreas urbanas onde cães domiciliados têm livre acesso a outras residências e a falta de monitoramento por parte dos órgãos públicos responsáveis pelo controle das populações de animais errantes podem interferir de forma positiva ou negativa na prevalência da dirofilariose. Estes fatores em sinergismo com aspectos sociais, econômicos e políticos determinam mudanças importantes no cenário desta zoonose. Alguns animais podem ainda apresentar baixo nível de infecção e mesmo não manifestar sinais e sintomas. Todos os cães e cadelas de quaisquer tamanhos ou raças são potencialmente passíveis de infecção.

No diagnóstico das filarioses caninas, a detecção e identificação das microfilárias são de grande importância para definir o agente específico. Resultados falso-positivos e falsonegativos podem levar a diagnósticos equivocados. Para minimizar este problema, as amostras de sangue avaliadas no presente trabalho foram submetidas simultaneamente ao exame direto de sangue total, Teste de Knott modificado e Snap Test para pesquisa de antígeno de Dirofilaria immitis.

O exame de sangue direto a fresco é o método mais comum e rápido para demonstrar a presença de microfilárias e pode ser utilizado na rotina das clínicas veterinárias que em geral possuem um microscópio óptico utilizado para outros exames. Para identificá-las, o Teste de Knott modificado é recomendado por Sloss et al. (29), sendo facilmente executável também em laboratórios. Em regiões endêmicas de Dipetalonema (Acanthocheilonema) reconditum (Grassi, 
1899), podem ser encontrados cães com microfilárias circulantes deste parasita, levando a diagnóstico equivocado de dirofilariose canina.

O município de Guaratuba representa para o estado do Paraná importante pólo turístico, com a população flutuante durante o período de alta temporada chegando a triplicar. $\mathrm{Na}$ medida do aumento da população são sentidos os efeitos da deficiência da estrutura urbana. Nestas condições, a vigilância preventiva é estratégica no sentido de não permitir a expansão das fronteiras de distribuição deste agravo à saúde de animais e que pode em certas circunstâncias atingir o homem, principalmente pela possibilidade de cães que são levados pelos responsáveis a infectarem-se e transportar esta zoonose para áreas indenes.

O nível de prevalência registrado neste trabalho de 0,94\% em relação ao índice de 5,4\% (8) pode estar próximo à realidade, pela utilização de todos os métodos clássicos para detecção de Dirofilaria immitis. Levando-se em consideração as limitações registradas no levantamento da população de cães, o município de Guaratuba apresenta reduzida circulação de Dirofilaria immitis. Todavia o município deve continuar a receber atenção especial das autoridades sanitárias estaduais e municipais, em relação a condições básicas de infra-estrutura, saneamento e prevenção de doenças, direcionadas a uma população sob permanente risco de agravos à saúde, com a finalidade de restringir ao máximo a contaminação ambiental e os riscos de infecção humana e canina. Igualmente, o controle e o rastreamento de cães errantes comprovam a importância, uma vez que estes animais geralmente são excluídos de quaisquer estatísticas envolvendo programas de sanidade animal.

\section{AGRADECIMENTOS}

Aos casais Sr. Romalino José Costa/Sra. Claudia Helena Xisto e Jesomir Uba Filho/ Rossana Cirio Uba pela amizade e fundamental colaboração no sucesso do presente trabalho.

\section{REFERÊNCIAS}

1. Ludlam KW, Jachowski LA, Otto GF. Potential vectors of Dirofilariaimmitis. J Am Vet Med Assoc. 1970;157(1-12):1354-1359.
2. Kasai N, Maltos EA, Costa JO. Dirofilaria immitis e Dipetalonema reconditum em cães de Vitória, Espírito Santo. Arq Esc Vet UFMG. 1981;33(3):425-429.

3. Labarthe N, Pereira NR, Soares AM, Bordin EL, Rotta A, Guerrero J. Prevalência de microfilárias de Dirophilaria immitis em diferentes populações caninas no Estado do Rio de Janeiro. Cães \& Gatos. 1989;27:24-25.

4. Labarthe N, Almosny N, Guerrero J, DuqueAraújo AM. Description of the ocurrence of canine dirofilariasis in the State of Rio de Janeiro, Brazil. Mem Inst O Cruz. 1997;2(1):47-51.

5. Ferreira AF, Barbosa FC, Mastrantonio EC. Ocorrência da dirofilariose canina na cidade de Uberlândia, MG, Brasil. Vet Notícias. 1999;5(1):57-61.

6. Fernandes CGN, Moura ST, Dias AR, Vieira Filho WS. Ocorrência de dirofilariose canina na região da Grande Cuiabá, Estado de Mato Grosso - Brasil. Braz J Vet Res An SC. 1999;36(5):258-261.

7. Suassuana ACD, De Paula VV, Feijó FMC. Ocorrência de cães parasitados por Dirofilaria immitis em Mossoró - RN. Rev Bras Med Vet. 2003;25(5/6):210-213.

8. Reifur L, Thomaz-Socol V, MontianiFerreira F. Epidemiological aspects of filariosis on the coast of Paraná state, Brazil: with emphasis on Dirofilaria immitis. Vet Parasitol. 2004;122:273-286.

9. Larsson MHMA, Meng MC, Recher JR, A. Estudo da variação da microfilare- mia de cães infestados por Dirofilaria immitis. Braz J of Vet Res An Sci. 1995;32(2):114-119.

10. Theis, JH. Public health aspects of dirofilariasis in the United States. Vet Parasitol. 2005;133:157-180.

11. Browne LE, Carter TD, Levy JK, Snyder PS, Johnson CM. Pulmonary arterial disease in cats seropositive for Dirofilaria immitis but lacking adult heartworms in the heart and lungs. Am J Vet Res. 2005;66(9):1544-1549. 
12. Echeto OEV, Simões D, Camacho JE, Oviedo ORV, Vale MO. Dirofilariosis em caninos: studio anatomopatológico de 15 casos. Rev Cient FCV-LUZ. 2005;15(5):406-411.

13. Atkins CE, De Francesco TC, Coats JR, Sidley JA, Keene BW. Heartworm in cats: 50 cases (1985-1997). J Am Vet Med Assoc. 2000;217(3):355-358.

14. Robinson NB, Chavez CM, Conn H. Pulmonary dirofilariasis in man: a case report and review of the literature. J Thorac Cardiovasc Surg. 1977;74:403-408.

15. Takeuchi T, Asami K, Kobayashi S, Masuda AM, Tanabe M., Miura S, Asakawa M., Murai T. Dirofilaria immitis infection in man: report of case of the infection in heart and inferior vena cava from Japan. Am J. Trop Med \& Hyg. 1981;305(5):966-969.

16. Shah M K. Human pulmonary dirofilariasis: review of the literature. South Med J 1999; 92(3):276-279.

17. Flieder DB, Moran CA. Pulmonary Dirofilariasis: A Clinicopathologic Study of 41 lesions in 39 patients. Human Pathol. 1999;30(3):251-256.

18. Cavallazzi RS, Cavallazzi AC, Souza IV, Cardoso JJD. Dirofilariose pulmonar humana: relato de sete casos. J de Pneumol. 2002;28(2):100-102.

19. Oshiro Y et al. Pulmonary dirofilariasis: computer tomography findings and correlation with pathologic features. J Comp Assist Tomog. 2004;28(6):796-800.

20. Asimacopoulos PJ, Katras A, Christie B. Pulmonary dirofilariasis* the largest singlehospital experience. Chest. 1992;102(3):851855.

21. Vital RJ, Mattos LA, Meirelles GSP. Human pulmonary dirofilariasis: atypical presentation of a rare disease. Rev Soc Bras Med Trop. 2006;39(1):94-95.
22. De Magalhães SPS. Descripção de uma espécie de filarias encontradas no coração humano, precedida de uma contribuição para o estudo de filariose de Wucherer e do respectivo parasito adulto, a Filaria bancrofti (Cobbold) ou Filaria sanguis hominis (Lewis). Rev Cur Prát Theor Fac Med RJ. 1887;3:129-215.

23. IBGE. Instituto Brasileiro de Geografia e Estatística. Contagem da população. 1:533. 1996. [cited 2005 May 20]. Disponível em:http://www.ibge.gov.br.

24. IPARDES. Instituto Paranaense de Desenvolvimento Econômico e Social. Zoneamento ecológico-econômico da APA de Guaraqueçaba. 1997. v. 2. [cited 2005 May 20]. Disponível em: http:// www.pr.gov.br/ipardes.

25. Granjean D. Enciclopédia do cão. Paris: ANIWA PUBLISHING; 2001. 635 p.

26. Newton WL, Wright, WH. The occurrence of a Dog Filariid other than Dirofilaria immitis in the United States. J Parasit. 1956; 42:246-256.

27. Serrão ML, Batista R, Paes-de-Almeida E, Santos P, Melo YF, Labarthe N. Descrição da população de animais de companhia e epidemiologia da dirofilariose canina em Itacoatiara, município de Niterói, R.J. Rev Bras Cien Vet. 2000;7(1):29-32.

28. Garcez LM, Fonseca-de-Souza N, Mota EF, Dickson LAJ, Abreu WU, Cavalcanti VFN, Gomes PAF. Focos de dirofilariose canina na Ilha de Marajó: um fator de risco para a saúde humana. Rev Soc Bras Med Trop. 2006;39(4):333-336.

29. Sloss MW, Kemp RL, Zajac A. M. Parasitologia clínica veterinária. $6^{a}$ ed. São Paulo: Manole; 1999. 198 p.

Recebido: 24/04/2006 Received: 04/24/2006

Aceito: $27 / 07 / 2006$ Accepted: 07/27/2006 